\title{
Synergistic effects of suberoylanilide hydroxamic acid combined with cisplatin causing cell cycle arrest independent apoptosis in platinum-resistant ovarian cancer cells
}

\author{
PEI-SHI ONG, XIN-QIAO WANG, HAI-SHU LIN, SUI-YUNG CHAN and PAUL C. HO \\ Department of Pharmacy, National University of Singapore, 18 Science Drive 4, Singapore 117543, Republic of Singapore
}

Received October 17, 2011; Accepted December 14, 2011

DOI: $10.3892 /$ ijo.2012.1354

\begin{abstract}
Histone deacetylase inhibitors (HDACIs) belong to an emerging class of anticancer compounds. It is increasingly recognized that their unique and complementary mode of action make HDACIs valuable agents in augmenting the cytotoxicity of conventional chemotherapeutics. We examined the potential for combined use of an approved HDACI, suberoylanilide hydroxamic acid (SAHA), with cisplatin (Cddp) in platinum-resistant ovarian cancer cells, OVCAR-3 and SKOV-3. The nature of the drug interaction following combinatory therapy was assessed using median effect analysis. We found that SAHA acted synergistically with Cddp over a wide range of concentrations in both cell types, resulting in favorable dose reductions of both compounds. In particular, in the more Cddpresistant SKOV-3 cells, more than 8-fold dose reduction of Cddp was achieved with the simultaneous use of SAHA and Cddp as compared to the dose required to elicit similar cell kill effects using Cddp alone. More importantly, therapeutic selectivity for ovarian cancer cells over normal fibroblast cells were maintained with the combinatorial therapy. We further observed that the augmentation of Cddp-induced cell death was mediated by the net increase in apoptosis and was independent of cell cycle arrest. Overall, concurrent application of SAHA and Cddp yielded the most favorable cell kill, indicating that this combination is promising for treatment of platinum-resistant ovarian tumors.
\end{abstract}

\section{Introduction}

Successful management of advanced epithelial ovarian cancer continues to be a challenge to clinicians. Despite the use of platinum-based chemotherapy for 30 years, the 5-year survival of patients with late-stage disease remains dismal. More than $70 \%$ of these patients succumb to the disease as a result of

Correspondence to: Dr Paul C. Ho, Department of Pharmacy, Faculty of Science, National University of Singapore, 18 Science Drive 4, Singapore 117 543, Republic of Singapore

E-mail: phahocl@nus.edu.sg

Key words: apoptosis, platinum, histone deacetylase inhibitors, drug synergism tumor recurrence and platinum chemoresistance (1). While a number of chemotherapeutic combinations have been tried as salvage therapies in the context of platinum chemoresistance, currently no regimens have shown remarkable success (1). Therefore, for patients with platinum refractory disease who are still desperately in need of more effective drug cocktails, continued evaluation of novel drug combinations that have the ability to re-sensitize the tumors to conventional chemotherapy is of crucial importance.

Histone deacetylase inhibitors (HDACIs) represent an emerging class of anticancer agents. HDACIs consist of diverse chemical structures including short-chain fatty acids, hydroxamic acid, cyclic tetrapeptides and benzamides (2). They were developed based on their ability to modulate chromatin dynamics through their inhibition of enzymes responsible for catalytic removal of acetyl groups in core nucleosomal histones (3-5). By doing so, these compounds favor uncoiling and relaxation of the chromatin structure, thus increasing the accessibility of transcriptional factors to the DNA structure. These result in the re-expression of silenced genes involved in cell cycle arrest (6), DNA repair (7) and apoptosis pathways (8). Such effects are translated into antitumor activities in a variety of cancer types (9), thereby highlighting the potential of HDACIs for widespread utility.

For ovarian cancer, HDACIs have shown tremendous promise in the treatment of this disease (10-15). In in vitro and in vivo ovarian cancer models, treatment with HDACIs resulted in growth inhibition, cell cycle arrest and apoptosis induction (10-15). In various cisplatin (Cddp)-resistant ovarian tumor cell lines, trichostatin A, a hydromaxic acid derived HDACI, induced $\mathrm{G}_{2}$-checkpoint arrest and apoptosis of these ovarian tumor cells through the induction of Bcl2-related Bad protein (16). More recently, Chao and colleagues showed that several HDACIs were able to reduce the viability of ovarian cancer cell lines with concomitant reduction of multidrug resistance-associated proteins 1 and 2, suggesting the potential additional benefits of these agents in ovarian cancer treatment (17).

Suberoylanilide hydroxamic acid (SAHA), a hydroxamic acid-derived HDACI, currently marketed under the brand name of Zolinza ${ }^{\mathrm{TM}}$ for the treatment of refractory cutaneous T-cell lymphoma (CTCL) $(18,19)$, is one of the most promising HDACIs identified in preclinical models of ovarian cancer (20). SAHA therapy in CTCL patients has not only yielded a good response rate but also clinically acceptable toxicity 
$(21,22)$, suggesting that it may be an attractive agent for use in combination therapy. With this preamble, we hypothesized that combined use of the novel combination of SAHA with Cddp can augment the cytotoxicity of Cddp and in turn overcome the chemoresistance of Cddp-resistant ovarian tumor cells to Cddp therapy. As postulated, this study showed that concurrent administration of SAHA and Cddp resulted in synergistic cell kill of ovarian tumor cells through an increase in cellular apoptosis. This drug combination retained selectivity for ovarian tumor cells and may be a promising therapeutic approach for treatment of Cddp-resistant ovarian disease.

\section{Materials and methods}

Reagents. Fluorescence diacetate (FDA), Cddp and SAHA were purchased from Sigma (St. Louis, MO), Pfizer (Bentley, Australia) and Alexis Biochemical Corp. (San Diego, CA), respectively. Triton X-100 was supplied by Bio-Rad (Hercules, CA). Fetal bovine serum (FBS), penicillin/streptomycin solution, trypsin/EDTA, Roswell Park Memorial Institute-1640 (RPMI-1640) medium and Dulbecco's Modified Eagle's medium (DMEM) were from HyClone Laboratories Inc. (Logan, UT). Propidium iodide (PI) was from Molecular Probes (Grand Island, NY).

Cell lines and treatment. Two ovarian cancer cell lines, OVCAR-3 and SKOV-3, that are resistant to clinically relevant concentrations of Cddp were used (ATCC; Manassas, VA). Lung fibroblast cell line, MRC-5, was used as the normal control (ATCC; Manassas, VA). OVCAR-3 cells were grown in RPMI1640 medium. SKOV-3 and MRC-5 cells were grown in DMEM. All cell cultures were supplemented with $1 \%(\mathrm{w} / \mathrm{v})$ penicillin/ strepto-mycin, $10 \%(\mathrm{v} / \mathrm{v}) \mathrm{FBS}$. Cells were grown at $37^{\circ} \mathrm{C}$ in a $5 \%$ $\mathrm{CO}_{2}$ humidified atmosphere.

Cddp was freshly diluted to required concentration from stock solutions before each experiment. Stock solution of SAHA was prepared in dimethyl sulfoxide (DMSO) at a concentration of $10^{-2} \mathrm{M}$ and stored at $-20^{\circ} \mathrm{C}$ until use. Vehicle control was used in all experiments.

Cell proliferation and cytotoxicity assay. Cell proliferation and cytotoxicity following drug treatment were assessed using fluorometric microculture cytotoxicity assay (FMCA) (23). Cells $(10,000)$ were seeded into each well of 96-well microtitre plates and allowed to adhere overnight. After that, the medium was replaced with $200 \mu \mathrm{l}$ of medium containing the test compounds or control medium. After $48 \mathrm{~h}$ of incubation, the plates were washed once with phosphate buffered saline (PBS). FDA solution $(200 \mu \mathrm{l})(2 \mu \mathrm{g} / \mathrm{ml})$ was added into the control, experimental and blank wells, then the plates were incubated for $30 \mathrm{~min}$ at $37^{\circ} \mathrm{C}$ and the fluorescence generated from each well was measured using a fluorescence plate reader, Spectrafluor (Tecan Instruments Inc., NC), equipped with a dichromatic mirror. The excitation $\lambda=485 \mathrm{~nm}$ and emission $\lambda=535 \mathrm{~nm}$ were used for all fluorescence measurements. The viability of each well was expressed as survival index (SI) which was defined as:

$$
\text { Survival index }(\mathrm{SI})=\frac{\mathrm{F}_{\text {drug-treated cells }}-\mathrm{F}_{\text {blank }}}{\mathrm{F}_{\text {control }}-\mathrm{F}_{\text {blank }}} \times 100 \%
$$

where by $\mathrm{F}_{\text {drug-treated }}$ cells represents fluorescence generated from drug treated wells; $F_{\text {control }}$ represents fluorescence generated from control untreated wells; $F_{\text {blank }}$ represents fluorescence generated from blank wells without cells.

$\mathrm{IC}_{50}$ value was defined as the drug concentration giving a SI of $50 \%$ of the control at the end of the treatment period. All $\mathrm{IC}_{50}$ values were obtained from survival curves using Graphpad Prism 4 Software (San Diego, CA).

Analysis of drug interaction. The median-drug effect analysis method by Chou and Taladay was used to evaluate the nature of interaction between SAHA and Cddp (24,25). Cells were seeded into a 96-well microtitre plate and allowed to adhere overnight. After which, the medium was replaced with either SAHA or Cddp alone or in combination in a fixed concentration ratio of each drug in increasing concentrations. A fixed $\mathrm{IC}_{50}$ concentration ratio of 1:4.35 and 1:1.90 was used for OVCAR-3 and SKOV-3 cells respectively. Cell proliferation after $48 \mathrm{~h}$ of incubation was assayed using FMCA as described previously.

Combination index (CI) at a series of values of fraction of cells affected $\left(f_{a}\right)$ was calculated to assess the degree of drug interaction $(24,25)$. For agents with mutually non-exclusive mode of drug action, the CI is calculated with the following equation:

$$
\mathrm{CI}=\frac{(\mathrm{D})_{1}}{(\mathrm{Dx})_{1}}+\frac{(\mathrm{D})_{2}}{(\mathrm{Dx})_{2}}+\frac{(\mathrm{D})_{1}(\mathrm{D})_{2}}{(\mathrm{Dx})_{1}(\mathrm{Dx})_{2}}
$$

where (D) $)_{1}$ and (D) $)_{2}$ are doses of Drug 1 (Cddp) and Drug 2 (SAHA) in combination that inhibit $\mathrm{x} \%$ of cell proliferation. $(\mathrm{Dx})_{1}$ and $(\mathrm{Dx})_{2}$ are the doses of Drug 1 (Cddp) and Drug 2 (SAHA) alone that also inhibit $x \%$ of cell proliferation $(24,25)$. $\mathrm{CI}<1$, indicates synergism; $\mathrm{CI}=1$, indicates an additive relationship, and $\mathrm{CI}>1$, indicates antagonism.

Following this, the dose reduction index (DRI) which determines the magnitude of dose reduction allowed for each drug in a synergistic combination as compared to the concentration of single agent required to achieve the same effect was also calculated as follows $(24,25)$ :

$$
(\mathrm{DR})_{1}=\frac{(\mathrm{Dx})_{1}}{(\mathrm{D})_{1}} \quad(\mathrm{DR})_{2}=\frac{(\mathrm{Dx})_{2}}{(\mathrm{D})_{2}}
$$

where (D) $)_{1}$ and (D) $)_{2}$ are doses of Drug 1 (Cddp) and Drug 2 (SAHA) in combination that inhibit $\mathrm{x} \%$ of cell proliferation. $(\mathrm{Dx})_{1}$ and $(\mathrm{Dx})_{2}$ are the doses of Drug 1 (Cddp) and Drug 2 (SAHA) alone that also inhibit $\mathrm{x} \%$ of cell proliferation $(24,25)$.

Analysis of apoptosis rate and cell cycle distribution. Apoptotic rate and cell cycle distribution were quantified by flow cytometry. Cells were treated with or without SAHA or Cddp alone or combination for $48 \mathrm{~h}$ in $25 \mathrm{~cm}^{2}$ flasks. After incubation, control and treated cells were harvested, washed with PBS and fixed in $70 \%(\mathrm{v} / \mathrm{v})$ ice-cold ethanol at $-20^{\circ} \mathrm{C}$ for at least $2 \mathrm{~h}$. Subsequently, cells were washed twice with PBS and incubated with PI/Triton X-100 staining solution ( $20 \mu \mathrm{g}$ of PI/ml of $0.1 \%$ (v/v) of Triton X-100 in PBS) containing $0.2 \mathrm{mg} / \mathrm{ml}$ of DNAse- 
A

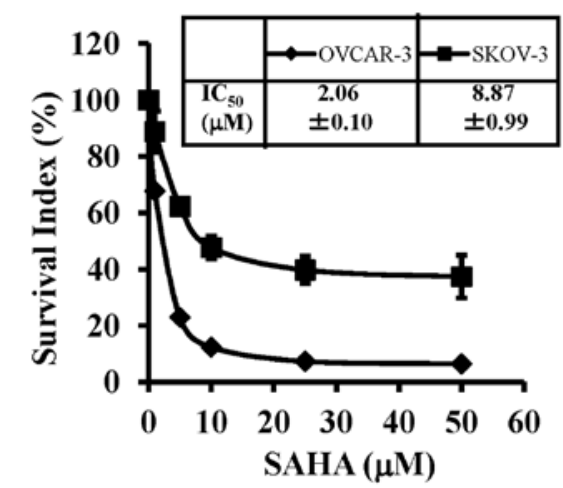

B

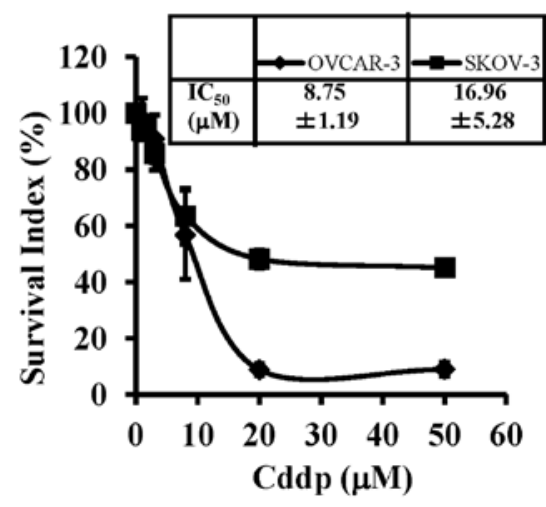

Figure 1. Dose-dependent cytotoxic cell kill and $\mathrm{IC}_{50}$ values of OVCAR-3 and SKOV-3 cells after (A) SAHA or (B) Cddp treatment. Cells $(10,000)$ were incubated with various concentrations of SAHA or Cddp for $48 \mathrm{~h}$. Cell viability was analyzed using FMCA. Survival index was calculated and presented as a mean $\pm \mathrm{SD}$ of three independent experiments. $\mathrm{IC}_{50}$ values were determined from the dose-response curve using GraphPad Prism 4 software.

free RNAse A for $30 \mathrm{~min}$ at $37^{\circ} \mathrm{C}$ in the dark. Data for at least 10,000 cells per sample was acquired using a flow cytometer (Beckman Coulter EPICS ${ }^{\circledR}$ ALTRA $^{\mathrm{TM}}$, Germany). DNA histograms obtained were analyzed using WINMDI Software (Scripps Institute, La Jolla, CA). Cells with DNA content less than the cells in the $G_{1}$ phase (sub- $G_{1}$ phase) were considered to be apoptotic (26).

Sequence-dependent interaction between SAHA and Cddp. Using OVCAR-3 cells as the prototype, SAHA and Cddp were applied in different sequential combinations to determine the effects of varied schedules on ovarian cancer cell death. Three different sequences of SAHA with Cddp were tested. They were: i) pretreatment with SAHA for $24 \mathrm{~h}$ followed by concurrent treatment with Cddp for $48 \mathrm{~h}$ (SAHA $\rightarrow$ Cddp), ii) concurrent treatment of SAHA with Cddp for $48 \mathrm{~h}$ (SAHA + Cddp) and iii) pretreatment with Cddp for $24 \mathrm{~h}$ followed by concurrent treatment with SAHA for another $24 \mathrm{~h}$ (Cddp $\rightarrow$ SAHA). For all sequences tested, total duration of Cddp treatment was kept at $48 \mathrm{~h}$. Cell survival was determined by FMCA. The reduction in cell survival for SAHA and Cddp combinatorial therapy versus treatment with Cddp alone was calculated for all three sequences used.

Statistical analysis. Statistical analysis was performed using Graphpad Prism 4 Software. Student's t-test was employed for
Table I. Dose reduction indices derived from cytotoxicity study of SAHA and Cddp combination in OVCAR-3 and SKOV-3 cells.

\begin{tabular}{lcccc}
\hline & \multicolumn{4}{c}{ Dose reduction index (DRI) } \\
\cline { 2 - 3 } & \multicolumn{2}{c}{ OVCAR-3 } & & \multicolumn{2}{c}{ SKOV-3 } \\
\cline { 2 - 3 } \cline { 5 - 6 } fa & SAHA & Cddp & SAHA & Cddp \\
\hline 0.1 & 0.88 & 1.23 & 1.18 & 8.01 \\
0.2 & 1.67 & 1.46 & 1.61 & 8.37 \\
0.3 & 2.55 & 1.64 & 1.97 & 8.57 \\
0.4 & 3.63 & 1.81 & 2.32 & 8.75 \\
0.5 & 5.00 & 1.97 & 2.71 & 8.91 \\
0.6 & 6.90 & 2.15 & 3.15 & 9.07 \\
0.7 & 9.79 & 2.67 & 3.72 & 9.26 \\
0.8 & 15.00 & 2.66 & 4.56 & 9.48 \\
0.9 & 28.56 & 3.16 & 6.19 & 9.84 \\
\hline
\end{tabular}

comparison between two groups and one-way ANOVA with Tukey's post hoc test was used for comparison between more than 2 groups. For these tests, a $\mathrm{p}<0.05$ was considered statistically significant.

\section{Results}

Cytotoxicity of Cddp and SAHA alone in OVCAR-3 and SKOV-3 cells. The in vitro cytotoxicity of SAHA or Cddp alone in both OVCAR-3 and SKOV-3 cells were first examined after $48 \mathrm{~h}$ of simultaneous drug treatment. Exposure to each drug resulted in significant growth inhibition and cytotoxicity in both cell lines in a concentration-dependent manner (Fig. 1). Cellular sensitivities for each drug were further illustrated by their $\mathrm{IC}_{50}$ values (Fig. 1). In OVCAR-3 cells, $\mathrm{IC}_{50}$ values for SAHA and Cddp were $2.06 \pm 0.10 \mu \mathrm{M}$ and $8.75 \pm 1.19 \mu \mathrm{M}$, respectively (Fig. 1). In SKOV-3 cells, $\mathrm{IC}_{50}$ values for SAHA and Cddp were $8.87 \pm 0.99 \mu \mathrm{M}$ and $16.96 \pm 5.28 \mu \mathrm{M}$ in chronological order (Fig. 1). The higher $\mathrm{IC}_{50}$ values of both drugs in SKOV-3 indicated a more drug resistant phenotype.

SAHA interacted synergistically with Cddp in OVCAR-3 and SKOV-3 cells. The effects of combined SAHA and Cddp on the survival of Cddp-resistant ovarian tumor cells were evaluated after $48 \mathrm{~h}$ of drug exposure. Increasing doses of Cddp and SAHA were applied to OVCAR-3 and SKOV-3 cells either alone or in combination. The doses of Cddp used for OVCAR-3 cells ranged from $2.24 \mu \mathrm{M}$ to $26.88 \mu \mathrm{M}$ whereas that for SKOV-3 ranged from $4.24 \mu \mathrm{M}$ to $50.88 \mu \mathrm{M}$. For SAHA, drug concentrations of $0.52 \mu \mathrm{M}-6.18 \mu \mathrm{M}$ were used for OVCAR-3 cells and concentrations of $2.22 \mu \mathrm{M}-26.61 \mu \mathrm{M}$ were used for SKOV-3 cells. As shown in Fig. 2A, combined use of SAHA and Cddp resulted in a greater inhibition of cell survival than either agents alone, at numerous doses, for both OVCAR-3 and SKOV-3 cells (Fig. 2A). Using the the median effect principles $(24,25)$, CI values of $<1$ was obtained for fa values $>0.4$ in OVCAR-3 cells (Fig. 2B). For SKOV-3 cells, the CI-fa curve indicated that SAHA and Cddp combination yielded a synergistic effect for fa values $>0.1$ (Fig. 2B). Favorable DRIs were also observed in both cell lines 
A
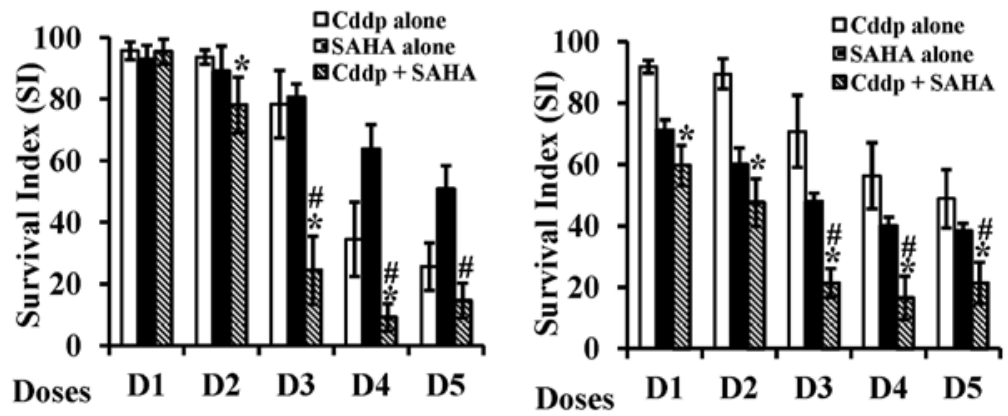

B

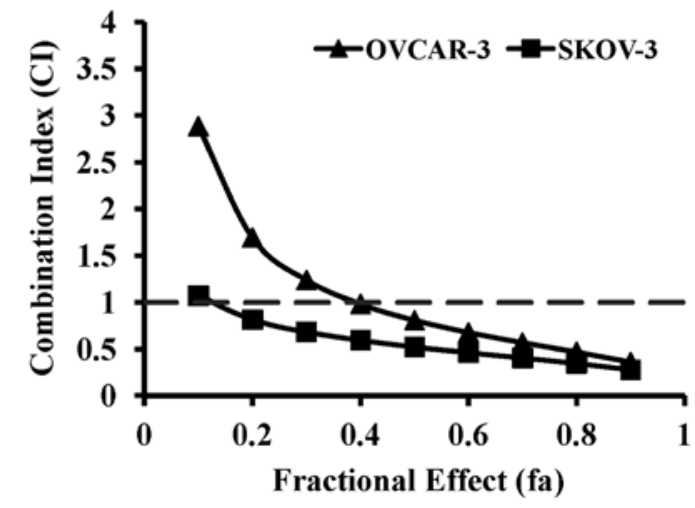

Figure 2. Effect of combined SAHA and Cddp treatment in OVCAR-3 and SKOV-3 cells. (A) Both cell lines were exposed to graded doses (D1-D5) of SAHA and Cddp alone or simultaneously for $48 \mathrm{~h}$. D1-D5 of Cddp were 2.24, 4.48, 8.96, 17.92 and 26.88 $\mu \mathrm{M}$ for OVCAR-3 cells. D1-D5 of SAHA were 0.52, 1.03, 2.06, 4.12 and $6.18 \mu \mathrm{M}$ for OVCAR-3 cells. For SKOV-3 cells, D1-D5 of Cddp were 4.24, 8.48, 16.96, 33.92 and 50.88 $\mu \mathrm{M}$. D1-D5 of SAHA for SKOV-3 cells were 2.22, $4.44,8.87,17.74$ and $26.61 \mu \mathrm{M}$. Cell survival was analyzed using FMCA. ${ }^{*}, \mathrm{p}<0.05$ for comparison between Cddp alone versus Cddp and SAHA and SAHA alone versus Cddp and SAHA respectively, using Student's t-test. All values are presented as mean \pm SD of three independent experiments. (B) Combinational indices were calculated using median effect analysis and plotted against fraction of cells affected.

(Table I). In OVCAR-3 cells, a 0.88-28.56-fold dose reduction was observed for SAHA upon combined use whereas that for Cddp ranged from 1.23- to 3.16-fold (Table I). Similarly, SKOV-3 cells showed positive DRIs ranging from 1.18 to 6.19 for SAHA and an even greater fold reduction of 8.01-9.84 for Cddp (Table I).

Cddp and SAHA synergistic combination showed therapeutic selectivity for ovarian cancer cells. To further establish the usefulness of this drug combination, the effects of this combination on a normal lung fibroblast cell line, MRC-5, was examined. When treated with Cddp alone for $48 \mathrm{~h}, \mathrm{IC}_{50}$ value of Cddp for MRC-5 cells was $>20 \mu \mathrm{M}$. When combined with SAHA at therapeutically relevant concentration of $2 \mu \mathrm{M}, \mathrm{IC}_{50}$ value of Cddp was reduced to $10.73 \pm 1.42 \mu \mathrm{M}$ (Table II). This is however still higher than that of $1.79 \pm 0.95 \mu \mathrm{M}$ and $5.89 \pm 1.99 \mu \mathrm{M}$ in OVCAR-3 and SKOV-3 cells, respectively, after concurrent Cddp and SAHA treatment (Table II). This therefore indicated preferential kill of tumor cells over normal controls using Cddp and SAHA combinatorial therapy.

Increased apoptotic cell kill with Cddp and SAHA combination compared to either drugs alone. We next examined whether the enhancement of cytotoxicity of Cddp by SAHA was associated with apoptosis induction. In OVCAR-3 cells,
Table II. IC $_{50}$ values of Cddp in OVCAR-3 and SKOV-3 cells after treatment with Cddp alone or concurrent treatment of Cddp with $2 \mu \mathrm{M}$ of SAHA.

\begin{tabular}{lccc}
\hline \multirow{2}{*}{ Treatment } & \multicolumn{3}{c}{$\mathrm{IC}_{50}$ values of Cddp $(\mu \mathrm{M})$} \\
\cline { 2 - 4 } & OVCAR-3 & SKOV-3 & MRC-5 \\
\hline Cddp alone & $8.75 \pm 1.19$ & $16.96 \pm 5.28$ & $>20$ \\
Cddp $+2 \mu \mathrm{M}$ SAHA & $1.79 \pm 0.95$ & $5.89 \pm 1.99$ & $10.73 \pm 1.42$
\end{tabular}

Data are expressed as the mean $\pm \mathrm{SD}$ of at least three independent experiments.

treatment with SAHA alone at $1 \mu \mathrm{M}$ resulted in $14.8 \%$ of apoptotic cells (Fig. 3A). With Cddp treatment alone at $3 \mu \mathrm{M}$ and $6 \mu \mathrm{M}$, percentages of apoptotic cells were $12.9 \%$ and $25.6 \%$, respectively. With concurrent SAHA at $1 \mu \mathrm{M}$ with either Cddp at $3 \mu \mathrm{M}$ or Cddp at $6 \mu \mathrm{M}$, apoptotic rates were significantly increased to $33.5 \%$ and $43.5 \%$, respectively (Fig. 3A). Similarly, for SKOV-3 cells, concurrent SAHA and Cddp therapy resulted in a significant increase in the percentage of apoptotic cells compared to treatment with either drug alone or the sum of the 
A

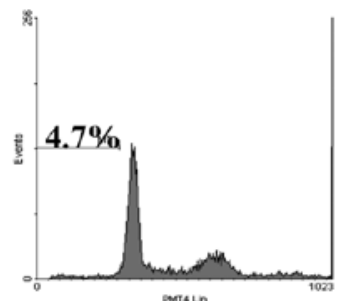

Control

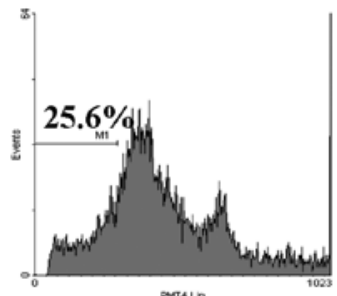

Cddp $6 \mu \mathrm{M}$ alone

B

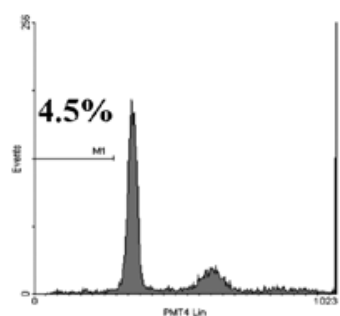

Control

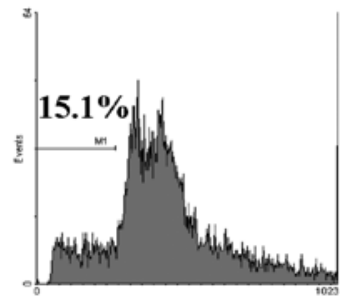

Cddp $6 \mu \mathrm{M}$ alone
OVCAR-3

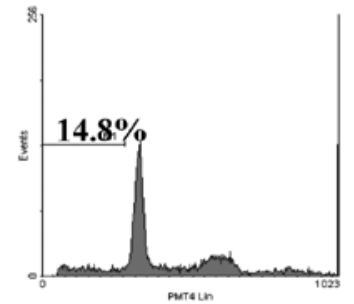

SAHA $1 \mu$ M alone

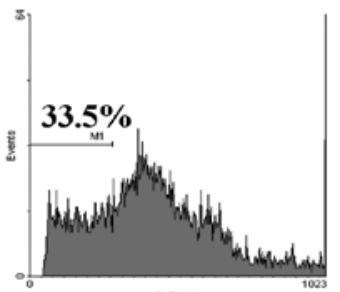

SAHA $1 \mu M$

+ Cddp $3 \mu \mathrm{M}$

SKOV-3

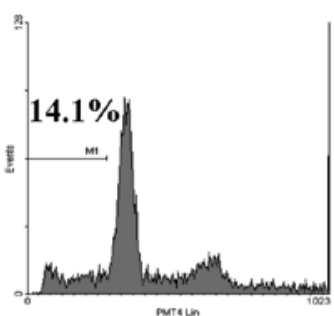

SAHA $2 \mu \mathrm{M}$ alone

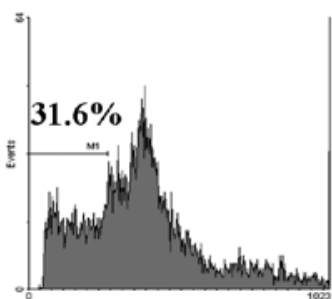

SAHA $2 \mu M$

+ Cddp $3 \mu \mathrm{M}$

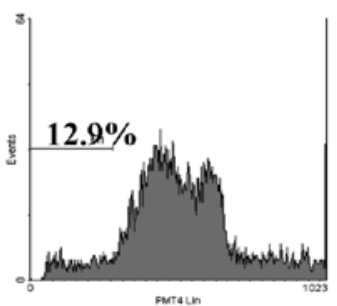

Cddp $3 \mu \mathrm{M}$ alone

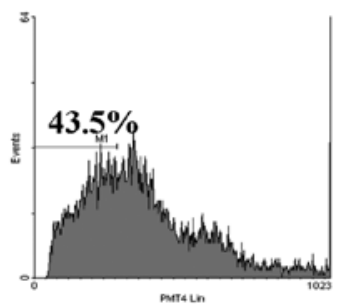

SAHA $1 \mu \mathrm{M}$

+ Cddp $6 \mu \mathrm{M}$

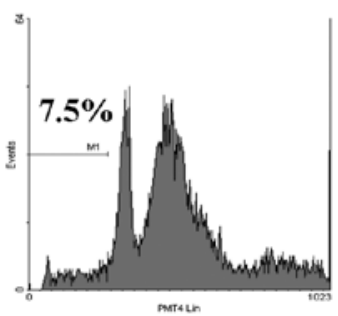

Cddp $3 \mu \mathrm{M}$ alone

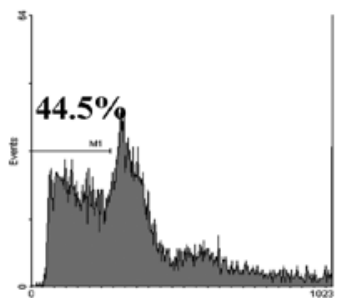

SAHA $2 \mu$ M + Cddp $6 \mu \mathrm{M}$

Figure 3. Apoptotic rate in (A) OVCAR-3 cells and (B) SKOV-3 cells after 48 h of treatment with SAHA or Cddp alone and in combination. Following 48 h of drug treatment, cells were harvested, fixed and stained with PI. Cell cycle profiles were captured using flow cytometry and analyzed using WinMDI software. Percentage of cells in sub- $\mathrm{G}_{1}$ phase which represents apoptotic cells was denoted in each DNA histogram.

apoptotic rates caused by the individual drugs (Fig. 4B). These data showed that combined SAHA and Cddp use resulted in synergistic apoptosis enhancement.

Effects of combined Cddp and SAHA therapy occurred independently of cell cycle arrest. To further elucidate if the cytotoxic potentiation of Cddp-induced cytotoxicity is also associated with alteration of cell cycle progession, we examined the cell cycle profiles of PI-stained cells using flow cytometry. Treatment with Cddp alone produced a consistent increase in cells arrested in the S-phase of the cell cycle in both ovarian cancer cell lines (Fig. 4, p<0.05) whereas SAHA alone did not reliably alter cell cycle progression at any particular phase (Fig. 4). With combined drug use, neither cell line displayed any significant perturbations of $G_{1}$ or $G_{2} / M$ phases of the cell cycle. In addition, DNA histograms analyzed showed a reduction of Cddp-induced S-phase arrest with co-administration of Cddp and SAHA (Fig. 4). These observations indicated that the net effect of concurrent drug use occurred independently of cell cycle arrest.

Different schedules influenced the outcome of Cddp and SAHA combination therapy. We assessed the effects of sequential administration of SAHA with respect to Cddp using three schedules namely: i) pretreatment with SAHA 
A
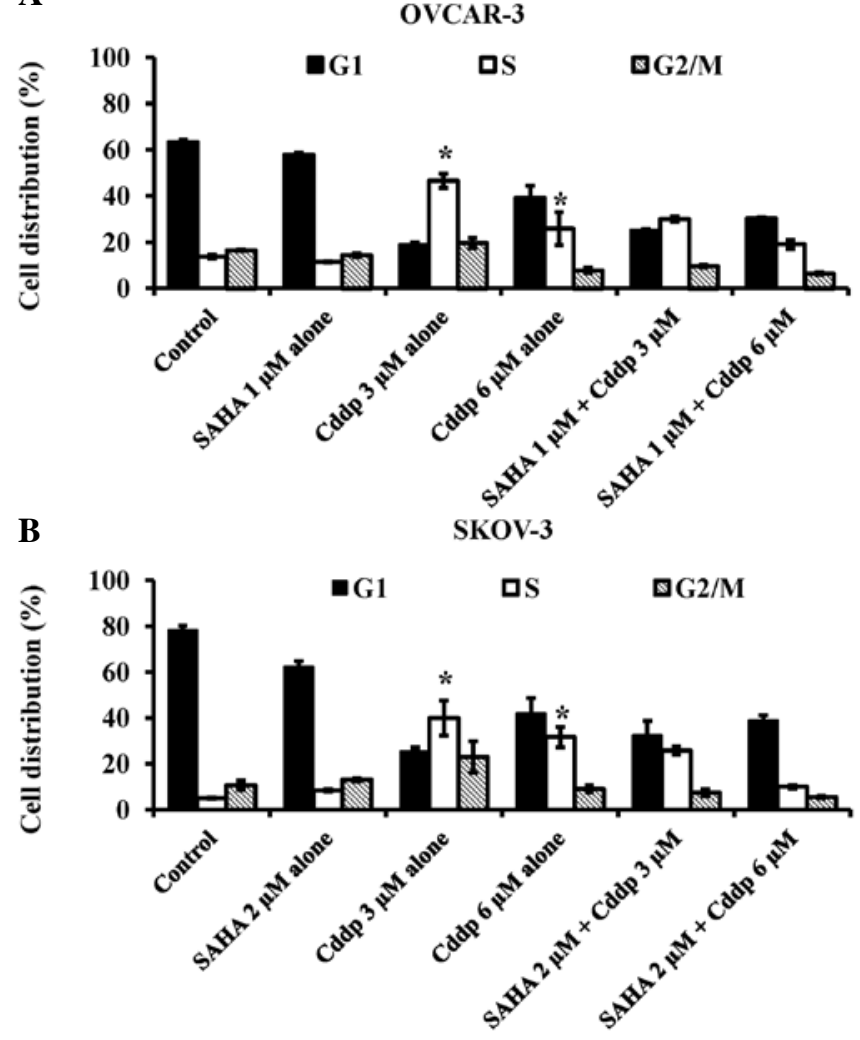

Figure 4. Cell cycle progression and arrest in (A) OVCAR-3 cells and (B) SKOV-3 cells after $48 \mathrm{~h}$ of treatment with SAHA or Cddp alone and in combination. Following drug treatment, cells were harvested, fixed and stained with PI. Cell cycle profiles were captured using flow cytometry and analyzed using WinMDI software. Each bar represents the mean \pm SD of at least two independent experiments ( $\mathrm{p}<0.05$ when compared with control using ANOVA with Tukey's post hoc comparison).

for $24 \mathrm{~h}$ followed by concurrent treatment with Cddp for $48 \mathrm{~h}$ (SAHA $\rightarrow$ Cddp), ii) concurrent treatment of SAHA with Cddp for $48 \mathrm{~h}$ (SAHA + Cddp), and iii) pretreatment with Cddp for $24 \mathrm{~h}$ followed by concurrent treatment with SAHA for another $24 \mathrm{~h}$ (Cddp $\rightarrow$ SAHA) (Fig. 5A). These schedules were designed to mimic possible clinical scenarios. From this experiment, the most cytotoxic schedule obtained was simultaneous treatment with SAHA + Cddp as illustrated by the greatest reduction in survival index (Fig. 5B). This is followed by pretreatment with SAHA for $24 \mathrm{~h}$ before concurrent treatment with Cddp (SAHA $\rightarrow$ Cddp) (Fig. 5B). Among the three schedules tested, pretreatment with Cddp for $24 \mathrm{~h}$ followed by concurrent treatment with SAHA (Cddp $\rightarrow$ SAHA) resulted in the least reduction in cell death (Fig. 5B). Similar results were obtained when either SAHA or Cddp were pre-incubated for a shorter duration of $4 \mathrm{~h}$ in sequential therapies (data not shown), indicating that concurrent treatment of SAHA and Cddp is the optimal dosing schedule for this chemotherapeutic combination.

\section{Discussion}

Concerted efforts to reverse platinum chemoresistance in ovarian cancer have yielded no acceptable drug cocktails of clinical utility. This continual lack of effective therapy continues to impede the clinical outcomes of patients with platinum
A

(i) SAHA $\rightarrow$ Cddp

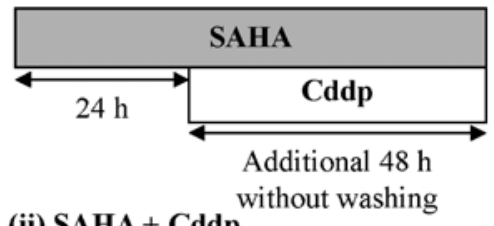

(ii) SAHA + Cddp

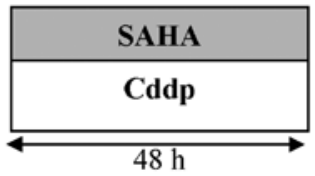

(iii) Cddp $\rightarrow$ SAHA

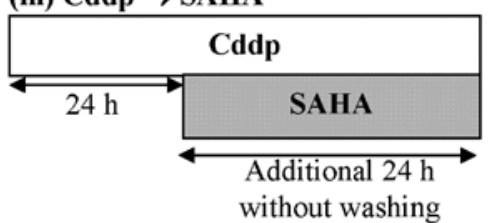

B

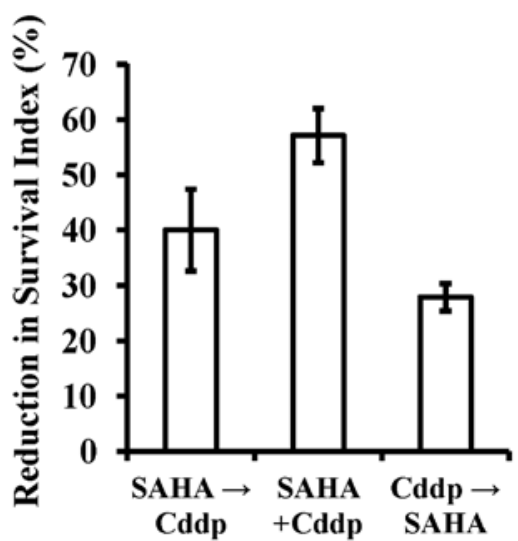

Figure 5. Effect of different sequence of drug treatment on the cell survival of OVCAR-3 cells. (A) Three different sequences of Cddp and SAHA exposure were employed. (i) Pretreatment with SAHA for $24 \mathrm{~h}$ followed by concurrent treatment with Cddp for $48 \mathrm{~h}$ (SAHA $\rightarrow$ Cddp); (ii) concurrent treatment of SAHA with Cddp for 48 h (SAHA + Cddp); and (iii) pretreatment with Cddp for $24 \mathrm{~h}$ followed by concurrent treatment with SAHA for $24 \mathrm{~h}$ (Cddp $\rightarrow$ SAHA). (B) OVCAR-3 cells were treated with $1 \mu \mathrm{M}$ of SAHA and $3 \mu \mathrm{M}$ of Cddp in three different schedules as described in (A). Cell viability and percent reduction in survival index were analyzed and data are presented as a mean \pm SD of 6-wells.

refractory ovarian carcinoma. Therefore, there continues to be an urgent need to search for new drugs combinations for this clinical scenario. In the present study, we investigated the strategy of using a commercially available HDACI, SAHA, to chemosensitize the antineoplastic effect of Cddp in two ovarian cancer cell lines, OVCAR-3 and SKOV-3. We provided the first demonstration that SAHA acted synergistically to increase the cytotoxic cell kill of Cddp in platinum-resistant ovarian tumor cells. Using the median effect analysis, we found that drug synergism was achieved over a wide range of Cddp and SAHA concentrations. This was in turn translated into favorable dose reduction of Cddp in both cell types. Another important significance of this combination is that normal cells were less susceptible to combinatorial treatment at therapeutically achievable concentrations of both SAHA 
and Cddp. It thus makes this novel combination a promising therapeutic strategy in the management of platinum-resistant ovarian carcinoma.

In this study, untransformed normal lung fibroblast cell line, MRC-5 instead of normal cell line from ovary was used as a surrogate control, as only immortalized normal ovarian surface epithelial cell lines are available from commercial tissue and cell line repositories such as American Tissue and Cell Culture, owing to the difficulty in maintaining the viability of untransformed normal ovarian cells in in vitro cell culture for prolonged period. Nevertheless, the adverse effects of chemotherapeutic drugs such as cisplatin and SAHA are not confined only to normal ovarian tissues when administered in usual chemotherapeutic regimens. For example, SAHA caused pulmonary embolism in 5\% of patients receiving the drug (22). With this understanding, the use of normal lung fibroblast as normal control in our experiments may also reflect the effects of such drug combination in nonovarian tissue.

Current interest in HDACI use has been increasingly focused on their potential in combinatorial therapy with a plethora of conventional chemotherapeutic agents, radiation or biological agents against different tumors (27-34). In ovarian cancer, combinatorial use of HDACIs has been investigated with traditional cytotoxic agents such as paclitaxel, docetaxel, Cddp and carboplatin (20). In particular for Cddp, HDACIs such as trichostatin A and valporic acid have shown potential for chemosensitization of Cddp-resistant ovarian cancer in preclinical models (33). Despite this positive finding, clinical translation of trichostatin A and valporic acid for augmentation of the cytotoxicty of Cddp is laced with difficulties. Therapeutic utility of trichostatin A is generally limited due to its poor bioavailability and toxic side effects at high doses (20). For valporic acid, recent Phase II study using magnesium valporate and hydralazine showed hematological toxicities to be the most debilitating adverse events that necessitated dose reduction in $60 \%$ of the study population (35). This may in turn result in the delivery of less than optimal doses of required chemotherapeutic agents and hence compromise the efficacy of this drug combination.

On the contrary, in a Phase IIb study of SAHA alone in patients with CTCL, combined grade 3-4 hematological toxicities were observed in only $5 \%$ of patients (22). In another study of SAHA in combination with carboplatin in non-small cell lung cancer, only $8 \%$ of patients discontinued therapy due to hematological toxicities (36). These studies thus imply that SAHA may possibly be better tolerated than valporic acid when used in combinatorial therapy clinically. Moreover, in preclinical studies, valporic acid was unable to show any synergistic cell kill with paclitaxel in SKOV-3 cells (33) whereas SAHA has been reported to show synergism when combined with paclitaxel in the more Cddp-resistant SKOV-3 cells (27). Collectively, this evidence suggests that SAHA has potentially wider applicability as a novel combinatorial agent either with Cddp alone or possibly with Cddp and paclitaxel, particularly in chemoresistant ovarian disease.

The ability of HDACIs to induce histone modification and subsequent transcription regulation suggest that the sequence with which HDACIs is being used as part of a chemotherapeutic cocktail with conventional antineoplastics is crucial for optimal anticancer effects $(37,38)$. In this study, pretreatment with SAHA followed by Cddp treatment did not result in greater cell kill compared with concurrent treatment with both agents. Drug treatment with SAHA alone or in combination with Cddp did not result in significant cell cycle arrest in ovarian tumor cells. This is in contrast with reports of disruption of cell cycle at the $G_{1}$ checkpoint by HDACIs through an increase in acetylated histone related proteins such as p21 (10,39). Moreover, with the benzamide class of HDACI as exemplified by MS-275, a more specific class of HDACI, drug synergism was not achieved with Cddp in SKOV-3 cells at therapeutically achievable concentrations of MS-275 (data not shown). Taken together, these results suggest that the enhancement of Cddp mediated cytotoxicity by SAHA in ovarian carcinoma cells may proceed through additional histone independent pathways which may be unique to SAHA and not other HDACIs. It further reinforced the distinctive properties of this novel combination that may not be generalizable to all HDACIs that are currently in various stages of development.

Increasing evidence suggests that the pan-HDACI, SAHA, acts on a diverse number of other molecular targets, independent of histone modification (40-42). In breast cancer cells, SAHA is able to acetylate unrecognized non-histone proteins, including transcriptional factors and regulators, chaperones, cell structure proteins, and glycolytic enzymes (42). With SAHA or other HDACIs, other histone independent effects such downregulation of phosphoAKT through reshuffling of protein phosphatase 1 complexes (43), induction of aberrant mitosis (44), inhibition of HSP90 function $(41,45)$, antagonism of the effects of androgen receptors (46) and upregulation of death receptor expression (47) have also been documented.

In studies of SAHA and Cddp combinatorial use in other tumor cell lines such as oral squamous cell carcinoma and cervical cancer, cytotoxic augmentation was mediated through a multitude of effects (48-51). For oral squamous cell carcinoma, Shen and colleagues showed that SAHA enhancement of Cddp-mediated apoptosis was brought about by an increased p53 activation, increased in pro-apoptotic BID, release of cytochrome $\mathrm{c}$ and activation of caspase-3 (48). In other separate study in oral squamous cell carcinoma, Rikiishi et al found that chemosensitization of Cddp by SAHA resulted in increased apoptosis via p53-independent activation of the mitochondrial pathway and caspase cascade that was accompanied by a decrease in cellular GSH levels (49). A more recent study in oral squamous cell carcinoma by Suzuki and colleagues revealed that drug synergism with SAHA and Cddp combination was propagated through a previously untapped mechanism such as endoplasmic reticulum stressmediated apoptosis that was independent of both calpain and c-Jun N-terminal kinase (50). SAHA augmentation of Cddp cytotoxicity has been reported in HeLa cells (51). In this cervival carcinoma cell line, the use of SAHA overcome platinum resistance through a decrease in antiapoptotic genes such as $\mathrm{Bcl}_{2}$, Bcl-xl, Mcl-1 and XIAP as well as accumulation of reactive oxygen species (51). These findings therefore highlighted that drug synergism brought about by SAHA on Cddp-mediated cytotoxicity is complex and multi-factorial. Currently, the molecular basis of this apoptotic enhancing effect of SAHA with Cddp in ovarian tumors is under investigation in our laboratory. Nonetheless, based on the present observations, combined use of SAHA and Cddp indeed represents a novel combination for consistent reversal of Cddp chemoresistance in ovarian carcinoma cells. 
In conclusion, our results showed that SAHA augmented the cytotoxic cell kill of Cddp in both OVCAR-3 and the more Cddp-resistant SKOV-3 cells, resulting in significant increase in cellular apoptosis. This unique combination has promising practical applicability that should be further investigated in future clinical studies.

\section{Acknowledgements}

This study was supported by research grants R148-000-032112 and R148-000-063-112 from the National University of Singapore. P.S. Ong is supported by NUS research scholarship.

\section{References}

1. Markman M: Pharmaceutical management of ovarian cancer: current status. Drugs 68: 771-789, 2008.

2. Bolden JE, Peart MJ and Johnstone RW: Anticancer activities of histone deacetylase inhibitors. Nat Rev Drug Discov 5: 769-784, 2006.

3. Drummond DC, Noble CO, Kirpotin DB, Guo Z, Scott GK and Benz CC: Clinical development of histone deacetylase inhibitors as anticancer agents. Annu Rev Pharmacol Toxicol 45: 495-528, 2005

4. Monneret C: Histone deacetylase inhibitors. Eur J Med Chem 40: 1-13, 2005.

5. Minucci S and Pelicci PG: Histone deacetylase inhibitors and the promise of epigenetic (and more) treatments for cancer. Nat Rev Cancer 6: 38-51, 2006.

6. Ogryzko VV, Hirai TH, Russanova VR, Barbi DA and Howard BH: Human fibroblasts commitment to a senescence-like state in response to histone deacetylase inhibitors is cell cycle dependent. Mol Cell Biol 16: 5210-5218, 1996.

7. Chen Z, Clark S, Birkeland M, Sung CM, Lago A, Liu R, Kirkpatrick R, Johanson K, Winkler JD and $\mathrm{Hu}$ E: Induction and superinduction of growth arrest and DNA damage gene 45 (GADD45) $\alpha$ and $\beta$ messenger RNAs by histone deacetylase inhibitors trichostatin A (TSA) and butyrate in SW620 human colon carcinoma cells. Cancer Lett 188: 127-140, 2002.

8. Marks PA, Richon VM and Rifkind RA: Histone deacetylase inhibitors: inducers of differentiation or apoptosis of transformed cells. J Natl Cancer Inst 92: 1210-1216, 2000.

9. Siegel D, Hussein M, Belani C, Robert F, Galanis E, Richon VM, Garcia-Vargas J, Sanz-Rodriguez C and Rizvi S: Vorinostat in solid and hematologic malignancies. J Hematol Oncol 2: 31-42, 2009.

10. Takai N, Kawamata N, Gui D, Said JW, Miyakawa I and Koeffler HP: Human ovarian carcinoma cells: histone-deacetylase inhibitors exhibit antiproliferative activity and potently induce apoptosis. Cancer 101: 2760-2770, 2004.

11. Takai N, Ueda M, Nishida M, Nasu K, Matsuda K, Kusumoto M and Narahara H: CBHA is a family of hybrid polar compounds that inhibit histone deacetylase, and induces growth inhibition, cell cycle arrest and apoptosis in human endometrial and ovarian cancer cells. Oncology 70: 97-105, 2006.

12. Takai N, Ueda T, Nishida M, Nasu K and Narahara H: A novel histone deacetylase inhibitor, Scriptaid, induces growth inhibition, cell cycle arrest and apoptosis in human endometrial cancer and ovarian cancer cells. J Mol Med 17: 323-329, 2006.

13. Takai N, Ueda T, Nishida M, Nasu K and Narahara H: M344 is a novel synthesized histone deacetylase inhibitor that induces growth inhibition, cell cycle arrest, and apoptosis in human endometrial cancer and ovarian cancer cells. Gynecol Oncol 101: 108-113, 2006.

14. Ueda T, Takai N, Nishida M, Nasu K and Narahara H: Apicidin, a novel histone deacetylase inhibitor, has profound anti-growth activity in human endometrial and ovarian cancer cells. Int J Mol Med 19: 301-308, 2007.

15. Qian X, LaRochelle WJ, Ara G, Wu F, Petersen KD, Thougaard A, Sehested M, Lichenstein HS and Jeffers M: Activity of PXD101, a histone deacetylase inhibitor, in preclinical ovarian cancer studies. Mol Cancer Ther 5: 2086-2095, 2006.

16. Strait KA, Warnick CT, Ford CD, Dabbas B, Hammond EH and Ilstrup SJ: Histone deacetylase inhibitors induce G2-checkpoint arrest and apoptosis in cisplatinum-resistant ovarian cancer cells associated with overexpression of the Bcl-2-related protein Bad. Mol Cancer Ther 4: 603-611, 2005.
17. Chao KC, Chang CC, Yen MS and Wang PH: Anti-tumor activity of histone deacetylase inhibitors and the effect on ATP-binding cassette in ovarian carcinoma cells. Eur J Gynaecol Oncol 31: 402-410, 2010.

18. Marks PA: Discovery and development of SAHA as an anticancer agent. Oncogene 26: 1351-1356, 2007.

19. Grant S, Easley C and Kirkpatrick P: Vorinostat. Nat Rev Drug Discov 6: 21-22, 2007.

20. Taki $\mathrm{N}$ and Narahara $\mathrm{H}$ : Histone deacetylase inhibitor therapy in epithelial ovarian cancer. J Oncol 2010: 458431, 2010.

21. Mann BS, Johnson JR, Sridhara R, Abraham S, Booth BP, Verbois L, Morse DE, Jee JM, Pope S, Harapanhalli RS, Dagher R, Farrell A, Justice R and Pazdur R: Vorinostat for treatment of cutaneous manifestations of advanced primary cutaneous T-cell lymphoma. Clin Cancer Res 13: 2318-2322, 2007.

22. Duvic M, Talpur R, Ni X, Zhang C, Hazarika P, Kelly C, Chiao JH, Reilly JF, Ricker JL, Richon VM and Frankel SR: Phase 2 trial of oral vorinostat (suberoylanilide hydroxamic acid, SAHA) for refractory cutaneous T-cell lymphoma (CTCL). Blood 109: 31-39, 2007.

23. Du YH and Ho PC: Arsenic compounds induce cytotoxicity and apoptosis in cisplatin-sensitive and -resistant gynecological cancer cell lines. Cancer Chemother Pharmacol 47: 481-490, 2001.

24. Chou TC and Talalay P: Quantitative analysis of dose-effect relationships: the combined effects of multiple drugs or enzyme inhibitors. Adv Enzyme Regul 22: 27-55, 1984.

25. Chou TC: Theoretical basis, experimental design, and computerized simulation of synergism and antagonism in drug combination studies. Pharmacol Rev 58: 621-681, 2006.

26. Darzynkiewicz Z, Bruno S, Del Bino G, Gorczyca W, Hotz MA, Lassota P and Traganos F: Features of apoptotic cells measured by flow cytometry. Cytometry 13: 795-808, 1992.

27. Cooper AL, Greenberg VL, Lancaster PS, van Nagell JR Jr, Zimmer SG and Modesitt SC: In vitro and in vivo histone deacetylase inhibitor therapy with suberoylanilide hydroxamic acid (SAHA) and paclitaxel in ovarian cancer. Gynecol Oncol 104: 596-601, 2007.

28. Rundall BK, Denlinger CE and Jones DR: Suberoylanilide hydroxamic acid combined with gemcitabine enhances apoptosis in non-small cell lung cancer. Surgery 138: 360-367, 2005.

29. Sonnemann J, Gange J, Kumar KS, Muller C, Bader P and Beck JF: Histone deacetylase inhibitors interact synergistically with tumor necrosis factor-related apoptosis-inducing ligand (TRAIL) to induce apoptosis in carcinoma cell lines. Invest New Drugs 23: 99-109, 2005.

30. Denlinger CE, Rundall BK and Jones DR: Proteasome inhibition sensitizes non-small cell lung cancer to histone deacetylase inhibitor-induced apoptosis through the generation of reactive oxygen species. J Thorac Cardiovasc Surg 128: 740-748, 2004.

31. Rundall BK, Denlinger CE and Jones DR: Combined histone deacetylase and NF-kappaB inhibition sensitizes non-small cell lung cancer to cell death. Surgery 136: 416-425, 2004.

32. Owonikoko TK, Ramalingam SS, Kanterewicz B, Baliu T, Belani CP and Hershberger PA: Vorinostat increases carboplatin and paclitaxel activity in non-small cell lung cancer cells. Int J Cancer 126: 743-755, 2010.

33. Lin CT, Lai HC, Lee HY, Lin WH, Chang CC, Chu TY, Lin YW, Lee KD and Yu MH: Valproic acid resensitizes cisplatin-resistant ovarian cancer cells. Cancer Sci 99: 1218-1226, 2008.

34. Camphausen K, Burgan W, Cerra M, Oswald KA, Trepel JB, Lee MJ and Tofilon PJ: Enhanced radiation-induced cell killing and prolongation of gammaH $2 \mathrm{AX}$ foci expression by the histone deacetylase inhibitor MS-275. Cancer Res 64: 316-321, 2004.

35. Candelaria M, Gallardo-Rincón D, Arce C, Cetina L, Aguilar-Ponce JL, Arrieta O, González-Fierro A, Chávez-Blanco A, De la Cruz-Hernández E, Camargo MF, Trejo-Becerril C, Pérez-Cárdenas E, Pérez-Plasencia C, Taja-Chayeb L, WegmanOstrosky T, Revilla-Vazquez A and Dueñas-González A: A phase II study of epigenetic therapy with hydralazine and magnesium valproate to overcome chemotherapy resistance in refractory solid tumors. Ann Oncol 18: 1529-1538, 2007.

36. Ramalingam SS, Maitland ML, Frankel P, Argiris AE, Koczywas M, Gitlitz B, Thomas S, Espinoza-Delgado I, Vokes EE, Gandara DR and Belani CP: Carboplatin and Paclitaxel in combination with either vorinostat or placebo for first-line therapy of advanced non-small-cell lung cancer. J Clin Oncol 28: 56-62, 2010. 
37. Marchion DC, Bicaku E, Daud AI, Richon V, Sullivan DM and Munster PN: Sequence-specific potentiation of topoisomerase II inhibitors by the histone deacetylase inhibitor suberoylanilide hydroxamic acid. J Cell Biochem 92: 223-237, 2004.

38. Sato T, Suzuki M, Sato Y, Echigo S and Rikiishi H: Sequencedependent interaction between cisplatin and histone deacetylase inhibitors in human oral squamous cell carcinoma cells. Int J Oncol 28: 1233-1241, 2006

39. Richon VM, Sandhoff TW, Rifkind RA and Marks PA: Histone deacetylase inhibitor selectively induces p21 ${ }^{\mathrm{WAF} 1}$ expression and gene-associated histone acetylation. Proc Natl Acad Sci USA 97: 10014-10019, 2000.

40. Kelly WK and Marks PA: Drug insight: histone deacetylase inhibitors - development of the new targeted anticancer agent suberoylanilide hydroxamic acid. Nat Clin Pract Oncol 2: 150-157, 2005.

41. Bali P, Pranpat M, Bradner J, Balasis M, Fiskus W, Guo F, Rocha K, Kumaraswamy S, Boyapalle S, Atadja P, Seto E and Bhalla K: Inhibition of histone deacetylase 6 acetylates and disrupts the chaperone function of heat shock protein 90: a novel basis for antileukemia activity of histone deacetylase inhibitors. J Biol Chem 280: 26729-26734, 2005.

42. Zhou Q, Chaerkady R, Shaw PG, Kensler TW, Pandey A and Davidson NE: Screening for therapeutic targets of vorinostat by SILAC-based proteomic analysis in human breast cancer cells Proteomics 10: 1029-1039, 2010.

43. Chen CS, Weng SC, Tseng PH, Lin HP and Chen CS: Histone acetylation-independent effect of histone deacetylase inhibitors on Akt through the reshuffling of protein phosphatase 1 complexes. J Biol Chem 280: 38879-38887, 2005.

44. Xu W, Perez G, Ngo L, Gui C and Marks PA: Induction of polyploidy by histone deacetylase inhibitor: A pathway for antitumor effects. Cancer Res 65: 7832-7839, 2005.
45. Marks PA, Miller T and Richon VM: Histone deacetylases. Curr Opin Pharmacol 3: 344-351, 2003.

46. Marrocco DL, Tilley WD, Bianco-Miotto T, Evdokiou A, Scher HI, Rifkind RA, Marks PA, Richon VM and Butler LM: Suberoylanilide hydroxamic acid (vorinostat) represses androgen receptor expression and acts synergistically with an androgen receptor antagonist to inhibit prostate cancer cell proliferation. Mol Cancer Ther 6: 51-60, 2007.

47. Insinga A, Monestiroli S, Ronzoni S, Gelmetti V, Marchesi F, Viale A, Altucci L, Nervi C, Minucci S and Pelicci PG: Inhibitors of histone deacetylases induce tumor-selective apoptosis through activation of the death receptor pathway. Nat Med 11: 71-76, 2005.

48. Shen J, Huang C, Jiang L, Gao F, Wang Z, Zhang Y, Bai J, Zhou H and Chen Q: Enhancement of cisplatin induced apoptosis by suberoylanilide hydroxamic acid in human oral squamous cell carcinoma cell lines. Biochem Pharmacol 73: 1901-1909, 2007.

49. Rikiishi H, Shinohara F, Sato T, Sato Y, Suzuki M and Echigo S: Chemosensitization of oral squamous cell carcinoma cells to cisplatin by histone deacetylase inhibitor, suberoylanilide hydroxamic acid. Int J Oncol 30: 1181-1188, 2007.

50. Suzuki M, Endo M, Shinohara F, Echigo S and Rikiishi H Enhancement of cisplatin cytotoxicity by SAHA involves endoplasmic reticulum stress-mediated apoptosis in oral squamous cell carcinoma cells. Cancer Chemother Pharmacol 64: 1115-1122, 2009.

51. Jin KL, Park JY, Noh EJ, Hoe KL, Lee JH, Kim JH and Nam JH: The effect of combined treatment with cisplatin and histone deacetylase inhibitors on HeLa cells. J Gynecol Oncol 21: 262-268, 2010. 